\title{
Study on the Relationship between the Right Hemisphere and Language
}

\section{Yulei Gao}

\author{
International Tourism Department, Nanjing Institute of Tourism and Hospitality \\ Nanjing, China \\ allen.0403@163.com
}

Keywords: right hemisphere; language competence; language learning

\begin{abstract}
It has long been accepted that the left hemisphere ( $\mathrm{LH})$ is the dominant hemisphere for language. Studies from cerebral lesion patients and normal subjects provide a large amount of evidence. While the current study sets out to explore the relationship between the right hemisphere (RH) and language. Reviewing some related literature, this paper finds that RH can also perform the function of lexical representations and process supprasegmental features, pragmatic and contextual information of language. The involvement of RH in language processing and comprehension can be affected by language status and learner factors. These findings are meaningful both for language instructors and learners.
\end{abstract}

\section{Introduction}

Language is specific to human beings. Children all over the world acquire their mother tongue at about the same time after they were born unless they are isolated during the critical acquisition years or unless they suffer from extreme external deficiency (Dai, Weidong, He Zhaoxiong: 2002). Even so, people can also lose their language when they suffer from cerebral lesions. Early in ancient Egypt before 3000BC, it was written from the Pharaonic medical papyrus: "If thou examinest a man having a smash in his temple, ...If thou callest to him (and) he is speechless (and) cannot speak...”. Though it was long recorded, they cannot uncover the relationship between human brains and language. Human brains were compared to "a dark box" before the use of functional brain imagining, and few evidence was obtained to explain the neural mechanism of language.

On April 12, 1861, Pierre Broca, a celebrated physician, was called to a patient named Leborgne with language impairment. Leborgne seemed to understand language but all he could speak was "tan". Broca obtained the authorization to autopsy his brain. During the procedure, he discovered a softening on a particular region, the posterior part of the left frontal lobe, at the third circumvolution where was afterwards named after as Broca's area (Broca, 1861). Then he reported his findings during the Parisian Anthropological Society, but attracted few attentions. After Leborgne, Broca followed eight patients who showed some kind of tissue injury in the same area with Leborgne. They could understand language but could not speak. These cases led Broca to conclude that the integrity of this area was necessary for the articulation of speech. In 1865, he declared to the public: "We speak with the left hemisphere". Since then, human brain was divided into a "major hemisphere", LH which is the language dominant hemisphere, and a "minor hemisphere". In the following 100 years, the exploration of the function of the RH didn't become a hot topic until 1950s. The author tries to review recent research on the language competence of $\mathrm{RH}$ and provide some implications for foreign language teaching.

\section{Language competence of RH}

$\mathrm{RH}$ and LH are symmetrical in appearance, but there is a great difference in functions. They can communicate with each other through a thick band of 200-500 million nerve fibers called the corpus callosum. LH is responsible for language, math and logic, while RH plays more of a role in spatial abilities, visual imagery and music. Even though, some language competence can be found in RH. 


\subsection{Lexical representation and $\mathrm{RH}$}

Lexical representation is a key question both in first and second language acquisition. Considering the topic under investigation, the author focuses his attention on the representation of lexicon in RH. As a dominant hemisphere for language, mental lexicon is mainly stored in the LH. RH, however, has also some kind of lexical representation. In his experiment, Day (1977) presented his 14 subjects (right-handed individuals with normal intact brains) 64 nouns (32 concrete nouns and 32 abstract nouns) and 64 nonwords (constructed by altering a single letter in each of the words) in each visual field. A manual reaction time (RT) measure was used to assess the relative efficiency of lateral stimulus-response pathways in processing linguistic information. The RT data indicated that a right visual field superiority for the speed of responses to abstract nouns but no difference between the left and right visual fields for the speed of response to concrete nouns. $\mathrm{LH}$ and $\mathrm{RH}$ were equally efficient at recognizing concrete object nouns while abstract nouns might be recognized only by LH. Therefore, we can safely conclude that RH at least has the representation of concrete nouns.

\subsection{Supprasegmental features and RH}

Generally speaking, patients with lesions in the RH cannot deal with supprasegmental features properly, such as tone, intonation and stress (Obler \& Gjerlow, 1999). Such patients tend to speak in a flat monotone. Blumstein and Cooper (1974) discovered that RH played more of a role in the syntactic function of the supprasegmental features in nontonal languages such as English. These findings are coincidence with RH being responsible for vocal tone, rhythm, and inflections, as well as their perception and pattern recognition.

\subsection{Context and RH}

As for information processing, $\mathrm{RH}$ tends to process the information holistically and nonverbally. Consequently, $\mathrm{RH}$ is good at processing languages with connotative meaning, metaphor, emotion and humor. Paradis (2004) also pointed out that lesions in RH caused such kind of difficulty.

\section{Influencing factors of RH involvement in language}

Both hemispheres possess the potential of language functions, especially before the completion of lateralization. Children suffered from large areas of cerebral lesions or hemispherectomy (either $\mathrm{LH}$ or $\mathrm{RH}$ ) can also acquire a language. The author will try to discuss several factors influencing the involvement of $\mathrm{RH}$ in language processing and comprehension. As foreign language learning research is attracting more attention, relating factors will be discussed here. Finally, complete content and organizational editing before formatting. Please take note of the following items when proofreading spelling and grammar:

\subsection{Language status}

As states in 2.2, some supprasegmental features are processed in RH. Guo Kejiao and Yang Qizhi (1995) also approached the distinction between the functions of two hemispheres in the cognition of the script, phonological and semantics of Chinese characters. Finally, they concluded that there was a "both-hemisphere effect" in the cognition of Chinese characters, and it is essentially related to the characteristics of the Chinese character itself. Cheung Mei-chun, Chan Yu-leung, et al. (2006) examined language lateralization of Chinese characters and English words associated with temporal lobe epilepsy (TLE) in Chinese-English bilinguals with left or right TLE, and discovered that normal controls demonstrates LH lateralization in reading English words but bi-hemispheric lateralization in reading Chinese characters. Therefore, the tonal inflection of Chinese spoken language may be processed to a greater extent in the RH than are nontonal languages such as English.

\subsection{Individual differences}

To a certain extent, language competence is different from individual to individual, especially for foreign language learners. Differences in individuals also lead to different degrees of $\mathrm{RH}$ involvement. 


\subsubsection{Sex}

In most cases, females generally have a better command of language than males. It is believed that there are more nerve fibers connecting two hemispheres for females than males. Physiologically, females become mature early than males, and they will reach a higher proficiency than males of the same age. Therefore, Females, as a group, are more likely than males to have bilateral language representation.

\subsubsection{Age of acquisition}

Age of acquisition is central to language acquisition studies. Concerning this question, Lenneberg pointed that there is a specific and limited time period for language acquisition, which is refer to as the Critical Period Hypothesis. Then what is the relationship between age of acquisition and RH involvement? The age of second language acquisition hypothesis (Genesee, Hamers, et al. 1978) pointed out: the closer in time that two languages of bilinguals are acquired, the more similar their lateralisation will be. Through their mata-analysis, Hull and Vaid (2006) concluded that early and late bilinguals demonstrated different patterns of lateralisation, with early bilinguals showing less lateralisation, and indeed, more bilateral involvement, relative to late bilinguals.

\subsubsection{Language proficiency}

Language proficiency is another issue in language acquisition of full attention. Different individuals with different language proficiency exhibit different neural mechanism. According to the stage hypothesis (Albert \& Obler,1978), early stages of L2 learning rely more on contextual cues to meaning, normally subserved by the $\mathrm{RH}$; however, with increasing proficiency in the language, a shift to LH dominance should ensue as syntactic and phonological aspects of L2 processing become more automatic. To test the hypothesis, Hull and Vaid (2006) reviewed concerning literature and concluded that nonproficient bilinguals may be $\mathrm{RH}$ lateralized, and that proficient bilinguals are indeed LH dominant, but only when the second language is acquired after the age of 6 . Consequently, the stage hypothesis is supported only for late bilinguals.

\section{Implications for foreign language education}

Few studies have approached foreign language teaching from a neurolinguistic perspective. An exception is Marcel Danei's (2003) educational construction of neurological bimodality, an attempt to find a neurological foundation for classroom language instruction. The underlying hypothesis is that there is a natural flow of information processing from the $\mathrm{RH}$ to the $\mathrm{LH}$ of the brain during language learning. Therefore, language instruction should follow the direction that the information flow from the $\mathrm{RH}$ to the LH. Though few evidence supports the hypothesis, some implications should be drawn from it. First of all, though female students tend to perform better in terms of bilateral language representation, no evidence supports that they will arrive at ultimate high proficiency. Therefore, there should be no discrimination between male and female students for the enrollment of students majoring in foreign languages. As for foreign language instructions, teachers should try different teaching methods and aids to help students master the language. For one reason, different hemispheres are responsible for different information processing, then the RH can be fully developed. For the other, it can attract students' attention in class. In addition, foreign language teachers should be aware of the differences between mother tongue and the foreign language, and provide students with authentic material to understand the pragmatic and contextual use of the language. Finally, second language learners can also arrive at native proficiency expect at the pronunciation level. Teachers should transform the knowledge of brain science into practical teaching methods to activate students' left and right hemisphere. They should also integrate students' hearing, vision, memory, emotion and reason to help students acquire a foreign language much easier. 


\section{Conclusion}

Previous studies have proved that $\mathrm{LH}$ is the dominant hemisphere for language. Four main language areas--Broca's area, Wernicke's area, supermarginal gyrus and angular gyrus--locate there. While the RH has also some language competence, and it can play a complementary role to some extent when the LH has a lesion. Reviewing some literature in this field, this paper conclude that $\mathrm{LH}$ performs the function of lexical representations and can process supprasegmental features, pragmatic and contextual information of language, and that both language status and learner factors can influence the involvement of $\mathrm{RH}$ in language mechanism. Neurolinguistics is a new field combining neuroscience and linguistics. If language instruction and learning will follow the disciplines, language learning will be less time-consuming and more fruitful.

\section{Acknowledgement}

In this paper, the research was funded by Jiangsu Provincial Office for Education Sciences Panning (Project No. C-a/2016/03/04).

\section{References}

[1] Dai, Weidong \& He Zhaoxiong. 2002, A New Concise Course on Linguistics for Students of English [M]. Shanghai: Shanghai Foreign Language Education Press 。

[2] Broca, P. P. 1861, Remarks on the seat of the faculty of articulate language followed by an observation of aphmia[J]. Reprinted in: Some papers on the Cerebral Cortex[C]. Translated by G. von Bonin. 1960. Springfield: Thomas.

[3] Day, James. 1977, Right-hemisphere language processing in normal right-handers[J]. Journal of Experimental Psychology: Human Perception and Performance. Vol.(3), 518-528.

[4] Obler, K. L. \& K. Gjerlow, 1999, Language and the Brain[M]. Cambridge University Press.

[5] Blumstein, S. \& Cooper, 1974, W. E. Hemispheric processing of intonation contours[J]. Cortex, (10)

[6] Paradis, Michel. 2004, A Neurolinguistic Theroy of Bilingualism[M]. Amsterdam: John Benjamins.

[7] Guo, Kejiao \& Yang Qizhi. 1995, ” Both -Hemisphere Effect” in the Cognition of Chinese Characters[J], Acta Psychologica Sinica (1).

[8] Mei-chun, Cheung, Yu-leung , Chan, et al. 2006, Language lateralization of Chinese-English bilingual patients with temporal lobe epilepsy: A functional MRI study[J]. Neuropsychology: Vol.(20).

[9] Genesee, F., Hamers, J. et al. 1978, Language processing strategies in bilinguals: A neurolinguistical study[J]. Brain and Language: Vol(5).

[10]Hull, Rachel \& Vaid, Jyotsna, 2006, Laterality and language experience[J]. Laterality (5)

[11]Albert, M., \& Obler, L.K. 1978, The Bilingual Brain: Neuropsychological and NeurolinguisticAspects of Bilingualism[M]. New York: Academic Press.

[12]Danesi, M., 2003, Second Language Teaching: A View from the Right Brain[M]. Kluwer Academic Publishers. 